\title{
Up Close: Department of Pure and Applied Chemistry at Strathclyde University, Glasgow S. Affrossman
}

This article is part of a series focusing on the research capabilities and goals of interdisciplinary laboratories pursuing materials research in universities, industry, and government.

Strathclyde University, nominally one of the newer universities, had its origins in 1796 when it was known as Anderson's Institution. Anderson hoped to provide in his will for the establishment of a university with faculties of law, divinity, medicine and arts. In fact, the funds only allowed, initially, for the appointment of one professor who taught philosophy, physics and chemistry. The inclination toward the practical disciplines was a continuing feature of the development of Strathclyde. Medicine came, and eventually went, but the closest approach to divinity was perhaps a onetime student, David Livingstone, the missionary explorer. The culmination of this selectivity was to be found in the title of the institution before university status was conferred in 1964, which was the Royal College of Science and Technology.

Though chemistry was taught from the inception of the institution, the first professor of chemistry, Thomas Graham, was only appointed in 1830 . He was an excellent choice, and his work on diffusion of gases, which made him internationally recognized, enhanced the University's reputation. One of his students was James Young, the founder of the oil industry, who extracted Scottish shale oil commercially, and who endowed a chair of technical chemistry to interact with the rapidly developing chemical industry.

The close relationship between the Strathclyde chemistry department and industry continues today with many and varied collaborative projects. With 40 members of staff, Strathclyde has one of the major chemistry departments in the United Kingdom, with a diversity of research interests. I shall describe only a few of the themes.

\section{Organic Crystals and Optoelectronics}

The limitations of communication with conventional electronics devices have led to a search for optical systems which can handle a vast increase in signal processing. The optoelectronic devices available are based on inorganic materials, such as lithium niobate, which can be grown highly pure, and possess the required crystal structures to give nonlinear optical properties.

Certain organic materials have also been shown to have nonlinear optical properties. Organics have the advantage, from a synthesis point of view, of allowing changes to be made in the chemical structure which may improve their performance. The key to using organic materials is the growth of very pure and good quality crystals. This is a difficult area of work, but over many years Prof. Sherwood and coworkers, Drs. Sheen and Roberts, have developed and refined the techniques of crystal growth to become one of the leading groups in the field. In parallel with the crystal growing, they have also developed characterization techniques which are essential for regulating the progress of the work.

Drs. Cruickshanks and Bailey, who have previous experience in determination of thermal conductivities by using thermal lens effects produced by intense laser radiation, have extended their work to the measurement of the interaction of laser light with the organic crystals.

A typical material is NMBA, 4-nitro, $4^{\prime}$ methyl benzylidene aniline. The size and quality of the crystals obtained from this compound can be judged from the photograph in Figure 1.

This compound, and similar materials, can double the frequency of light which is transmitted through the crystal. Figure 2 shows green light (as a circle) emitted from an m-nitro aniline crystal irradiated with invisible infrared radiation.

Viable commercial application of such optical devices requires both high efficiency of the frequency conversion and a good resistance of the compound to the damaging effect of the incident radiation. The extent of damage can be measured by $x$-ray topography, using the SERC synchrotron facility, another technique in which the team has a long-standing interest.

The organic materials lend themselves to tailoring of the molecular structure to optimize the nonlinear properties. The above research is backed by extensive theoretical work, under the guidance of Dr. Pugh, to calculate the properties of the compounds with various molecular and crystal structures.

Modification of organic crystal structure can be profitable even with less exotic problems. Prof. Sherwood's group has devised crystal growth modifiers which delay diesel oil freezing as the temperature is lowered. This is of much interest to the oil companies, and also to everyone who has waited in vain for a bus on an exceptionally cold morning.

The many processes in the modern chemical and pharmaceutical industries which involve precipitation of crystalline materials are providing new lines of research in this important aspect of material handling.

\section{Polymers}

Polymer science is one of the widest areas of research in the department. The interests cover synthesis, physical properties, structure, fibers and rheology, and surface properties, and much of the work is directed to materials aspects.

Prof. Pethrick has developed materials for use as electron beam resists. A series of promising materials, comb structures, have arisen from his group's work on solvent-polymer interactions and electron beam irradiation studies.

Recent interest in chemical sensors stimulated interaction between the polymer group and the organic chemists, with expertise in enzyme technology to develop a new type of very specific sensor which is simple to operate.

Another development from this group is a versatile but inexpensive instrument for measuring rates of cure of filled or simple polymer systems, which will be offered for sale by an instrument manufacturer. The instrument will be invaluable in studying the many technological problems involv- 


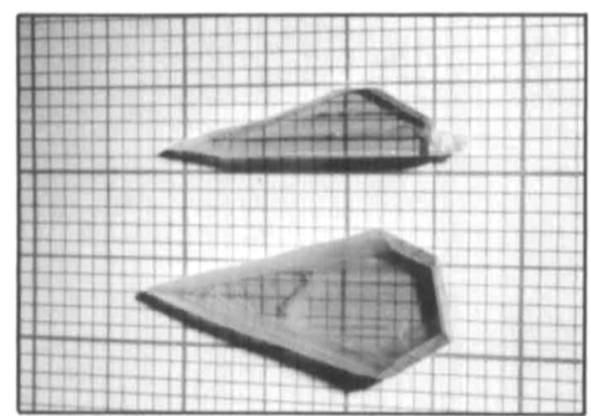

Figure 1. Size and anality of crystals offninct from NMBA, 4-nitro, 4'-methyl benzylidene aniline.

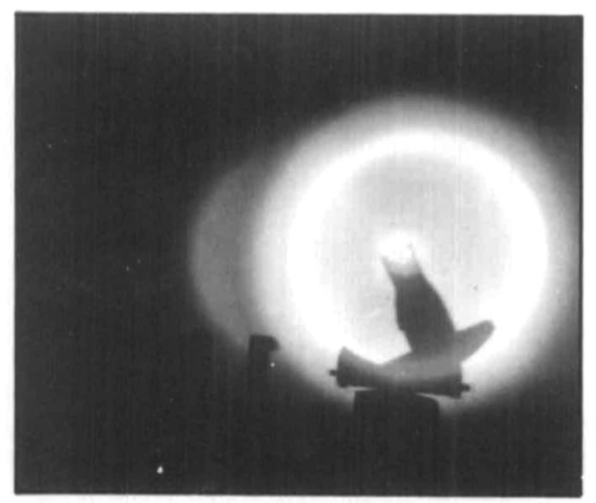

Figure 2. Grem light (as a circle) colnitted from an m-nitro aniline crystal irradiated with invisille infrared mdiation.

ing filled polymers

Attachment of specific reactive functionalities to polymers is the specialty of Prof. Sherrington. Some polymers are modified to produce phase transfer catalysts, and others more simply to attract certain ions or molecules from liquids for use in extraction or purification. There are obvious environmental reasons for removal of dilute concentrations of pollutants. Concentration of ions of valuable metals by adsorption before further processing can make a process become economically viable.

The opposite phenomenon, keeping molecules within the polymer until they eventually diffuse out, has become an important mechanism for controlled drug release in medicine. Prof. Graham has used his acadenic expertise to set up a company to produce a special range of hydrogels with the required characteristics for biocompatability and drug capacity. The slow, steady release of the drugs can be much more efficient medically than spasmodic dosage, and less stressful to the patient. Prof. Graham has worldwide backing for his work.

Dr. Ferguson is an international authority on polymer rheology, and he has developed an on-line process control rheometer. Several major companies are interested in its potential for automated control of process plants.

A particular interest of Dr. Ferguson is template polymerization, the use of one macromolecule to control the synthesis of a second. This subject merges kinetics and rheology. The effect of rheology on polymer behavior is strikingly shown in Figure 3 . Elongational flow of a polymer solution gives orientation of the polymer molecules leading to phase separation.

Dr. Richards uses scattering techniques to determine molecular and supramolecular structure of polymer systems, especially block copolymer and interpenetrating network phase separated systems. Information on these materials is obtained from small angle $x$-ray, or neutron, scattering. The block copolymers can exhibit considerable long-range order (see Figure 4) whereas the interpenetrating networks have no long-range order though they have phase separation.

The latter are considerably tougher than the original single polymers. An important area of the work is the study of the effect of bulk extension on the deformation of the elastomeric molecules.

A new technique, utilized by Dr. Richards, is the reflection of neutrons from polymers at air-liquid, liquid-liquid, and liquid-solid interfaces. This technique can give unique information on the structure at the interface at the $10 \mathrm{~nm}$ level

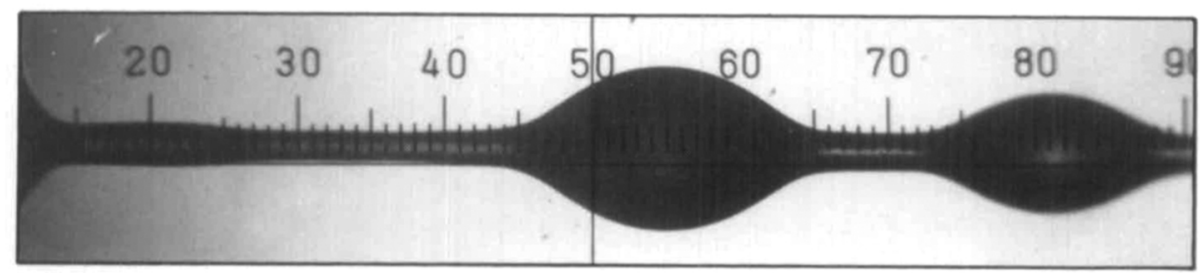

Finge 3. Droplets formed after chonsational flowe of a polymer solution Birefringence effects indicate orientation of the polymer molecules lading to phase scparation. (Photo taken using polarized light.)

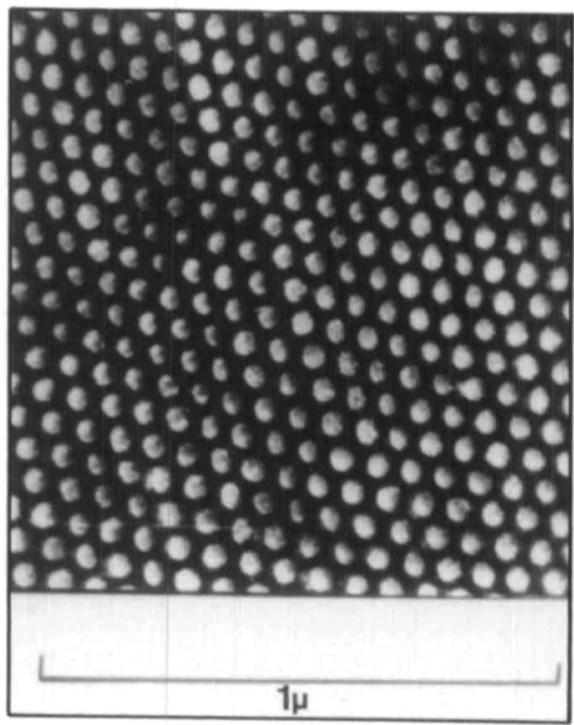



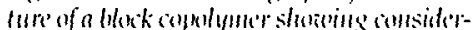
able lons-mist' ontit:

\section{Thin Film Coatings}

The latest holder of the chair of technical chemistry, Prof. Hitchman, maintains the relationship between the department and the forefront of industrial research. His group is developing reactors for production of very uniform layers of epitaxial silicon by means of chemical vapor deposition for use in microelectronic fabrications. The project relies on the understanding of the kinetics and mechanisms of chemical vapor deposition processes, which have resulted from Prof. Hitchman's recent rescarch.

It is appropriate to end with the most exciting recent technological breakthrough, high T, superconductors. Prof. Hitchman is developing chemical vapor deposition systems for preparing thin films of these promising materials.

Stanley Affrossman

Department of Pure and Applied

Chemistry

Strathclyde University

Glasgow GI IXI

Scotland

Telephone 041-552-4400, ext. 2299/2288

Stanley Affrossman gmduated BSC and PhD from the Uminersity of Clasgere. He spent treo years in Canata at the National Researd Contucil in Ottazea, then returned to Glasume to the then Royal College of Science and Tochnology in 1963. An carly concern zeith heteregeneous catalysis led to a zonder interest in surface analysis, and nore he Use's XPS and SIMS fo stindy pmblems rilated to polymer surfaces, polymer-filler inlerfaces, and conthlysts. 